\title{
Theoretical Modeling and Computational Simulation of Electronic Properties of Nanomaterials
}

\author{
Shaogang Hao, ${ }^{1}$ Zhirong Liu, ${ }^{2}$ Junqiang $\mathrm{Lu}^{3}$ and Gang Zhou ${ }^{4}$ \\ ${ }^{1}$ The Ames Laboratory of USDOE and Department of Physics, Iowa State University, Ames, IA 50011, USA \\ ${ }^{2}$ College of Chemistry and Molecular Engineering, Peking University, Beijing 100871, China \\ ${ }^{3}$ Department of Physics, University of Puerto Rico, Mayaguez, PR 00681, USA \\ ${ }^{4}$ Department of Physics, Tsinghua University, Beijing 100084, China \\ Correspondence should be addressed to Shaogang Hao, sghao@ameslab.gov
}

Received 20 June 2011; Accepted 20 June 2011

Copyright (c) 2011 Shaogang Hao et al. This is an open access article distributed under the Creative Commons Attribution License, which permits unrestricted use, distribution, and reproduction in any medium, provided the original work is properly cited.

Over the past two decades, the emergence of new techniques has made synthesis and construction of matters at nanoscale possible, leading to an extremely rapid rise of the field of nanomaterials science. For instance, today's electronics industry is the result of advances in nanomaterials research. As electronic devices get smaller, there are increasing challenges in silicon technology. Materials with new scaling properties need to be developed urgently. Indeed, nanomaterials science promises a wide range of novel applications of new perspectives.

Meanwhile, theoretical modeling and computational simulation have advanced as much as the experimental techniques in nanomaterials science. The development ranges from density functional algorithms to mesoscale methods, which enables the pursuit of the characteristic nature of nanomaterials as well as predict and design functional nanomaterials. On the other hand, recent development of parallel processor supercomputers has led to tremendous increase (by a factor of a million) in computational power. These developments together have been creating new opportunities for modeling and simulation of electronic properties of nanomaterials, as well as for designing novel nanoelectronic devices. In this special issue, six papers are selected to provide discovery of novel phenomena, fundamental understanding and prediction, and design of nanomaterials from zerodimensional nanoparticles, to one-dimensional graphene ribbons and carbon nanotubes, to two-dimensional films.

The first paper of this special issue introduces a novel method to synthesize Ag-Au nanoparticles for the purpose of potential application as catalysis in chemical industries.
Size, shape, and some other basic properties of the resulting bimetallic Ag-Au nanoparticles have been characterized by measurements and computer simulations.

The second paper of this special issue studies the intrinsic spin-orbit coupling effect in the band structures of graphene nanoribbons. Graphene's fascinating properties mostly originate in its simply beautiful electronic structure. Due to the exceedingly small effective mass, graphene holds the promising potential of next generation semiconductor industry. Therefore, enormous attentions are paid to the band structure of graphene. Using tight-binding model and state-of-the-art $a b$ initio simulations, it has been discovered that a band gap might be opened at the Dirac point in graphene nanoribbons due to the spin-orbit coupling. Moreover, the ribbon width and edge configuration are found to play an important role in modulating the spin-orbit coupling strength.

The third paper reports the fabrication of silica films with mesoporous. The size of the mesoporous is quite uniform. and the X-ray diffraction shows that the mesoporous pattern in the films is highly ordered. Such interesting structure could be applied as template for further synthesis of quantum devices.

The fourth paper proposes two designs of silicon-onInsulator (SOI) structures by theoretical modeling. Performances of these SOI structures have been systematically studied in detail. It is interesting that when the film thickness shrinks to nano-/subnano-scale, the electrical behaviors become very distinct compared with classical cases, which could be used to design new SOI-based devices. 
The fifth paper reports the synthesis of $\mathrm{Cu} / \mathrm{SnO}_{2}$ nanoparticle/Cu sandwich structure and studies the electric performance of the products. Armed with theoretical modelings, the authors demonstrated that when the dielectric goes to the size of nanoscale, the conduction behavior is dominated by the Poole-Frenkel mechanism.

The last paper simulates the $\mathrm{TiO}_{2}$-nanoparticle encapsulated single-walled carbon nanotube $\left(\mathrm{TiO}_{2}\right.$-NP@SWCNT) by using the Lennard-Jones potentials. Such novel "peapod" nanostructures have been holding a lot of attention in both theoretical and experimental aspects. $\mathrm{TiO}_{2}$ is a well-known functional material which is widely used in chemical and environmental industries, due to its great catalytic performance. This paper discusses the mechanism of encapsulating $\mathrm{TiO}_{2}$ nanoparticles into a one-dimensional container, carbon nanotubes. It is shown that $\mathrm{TiO}_{2}$ particle needs to overcome a strong barrier to enter the nanotube from tube mouth.

Shaogang Hao Zhirong Liu Junqiang Lu Gang Zhou 

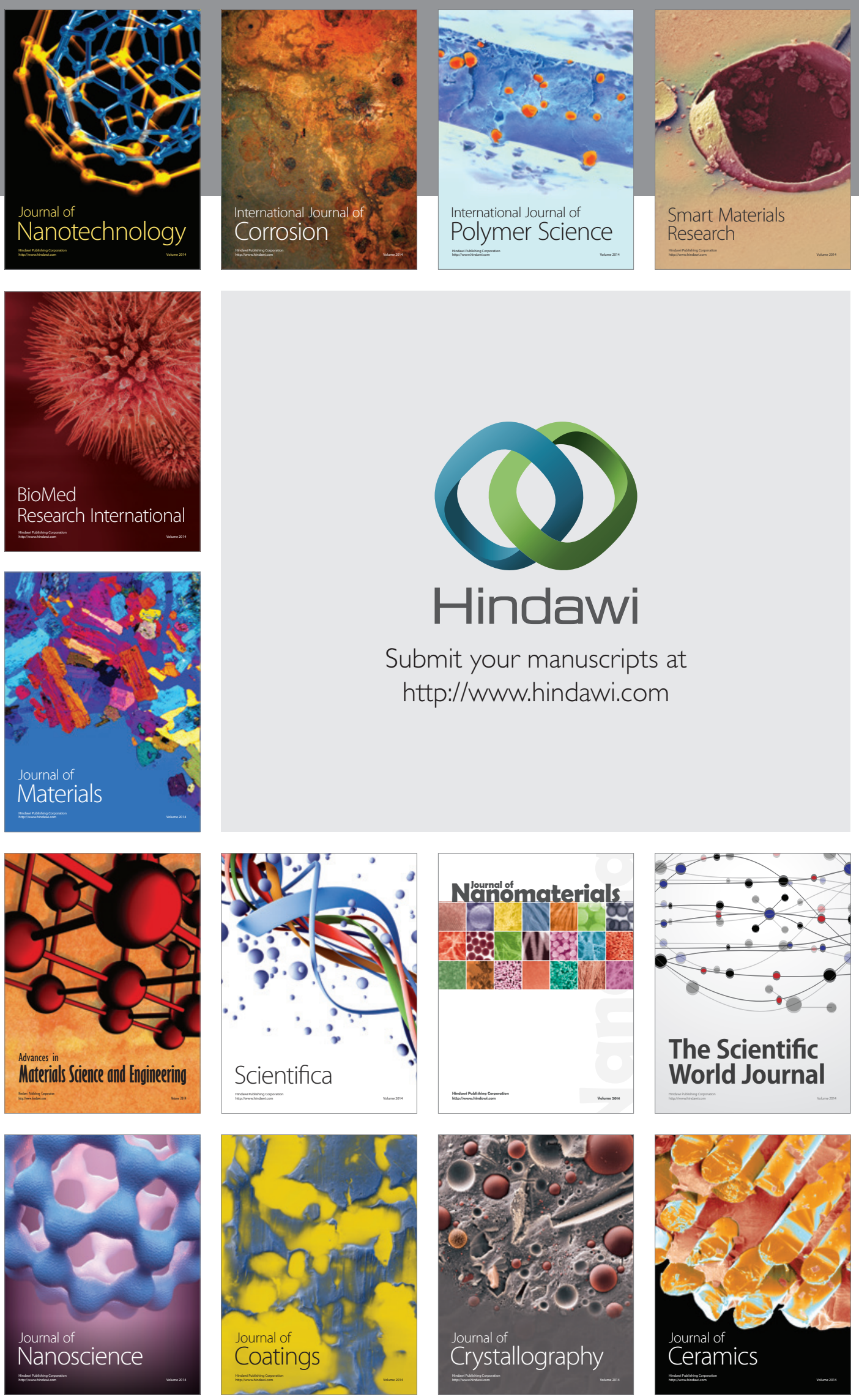

The Scientific World Journal

Submit your manuscripts at

http://www.hindawi.com

\section{World Journal}

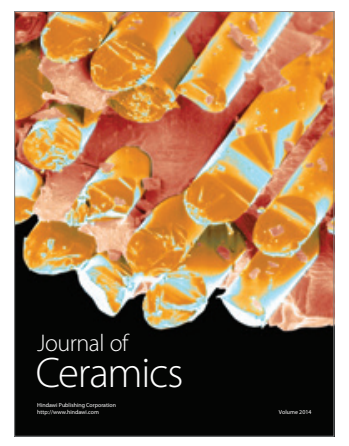

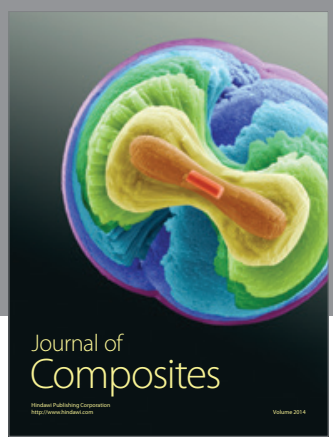
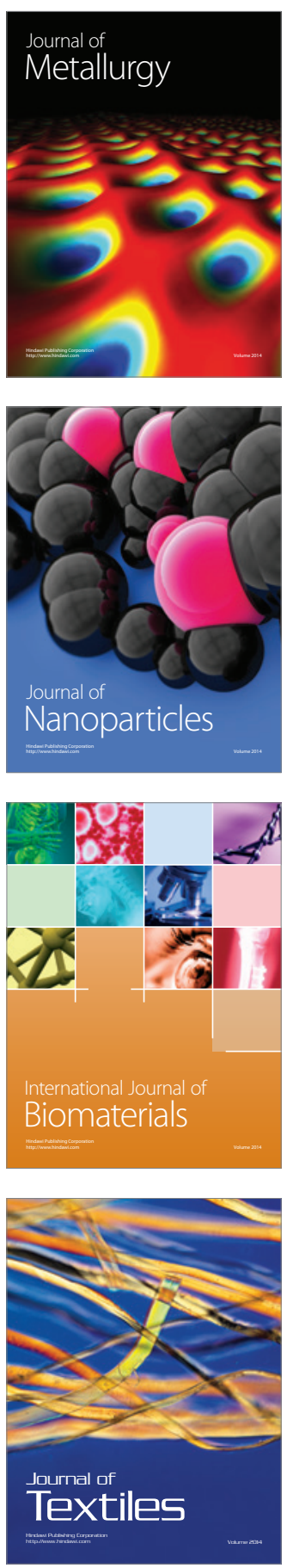